\title{
Fuzzy Real Option Analysis for IT Investment in Nuclear Power Station
}

\author{
Chen Tao, Zhang Jinlong, Liu Shan, and Yu Benhai \\ School of management, Huazhong University of Science and Technology, \\ Wuhan, 430074, China \\ chentaohust@yahoo.com.cn, jlzhang@mail.hust.edu.cn, \\ liushan@163.com, ybhai@163.com
}

\begin{abstract}
This paper develops a comprehensive but simple methodology to valuate information technology investment in a nuclear power station based on fuzzy risk analysis and real option approach. By linking the variability of expected payoffs to specific sources of risk factors, this method could help decision makers achieve a more reliable valuation of the target investment under multiple sources of risk, and avoid complex estimation tasks at the same time. The proposed approach can be easily adopted to value any irreversible investment subject to a high degree of uncertainty that is common in the field of energy economics.
\end{abstract}

Keywords: information technology investment, real options analysis, risk assessment, nuclear industry.

\section{Introduction}

Real options analysis (ROA) has been accepted as a modern approach for risk investment analysis. In recent years, this approach has been rapidly evolving, and is now spreading to the field of energy economics. It is ideally suited for the valuation of investments in tangible assets and infrastructure like energy generation plants that are subject to a high degree of uncertainty. Similar to energy investment, information technology (IT) investment is characterized by the nature of Long-time horizons, significant risks and irreversibility. Researchers propose to introduce ROA to IT investment decision-making. Benaroch and Kauffman illustrated the use of real options techniques in the context of a decision to expand a banking ATM network [1]. Taudes suggested that the value of IT investment can be defined as the sum of economic value and option value [2]. L. milder et al. use ROA to value an IT infrastructure investment in South Korea [3].

However, several challenging preliminary requirements has prevented the application of real options theory in practice. For example, Black-Scholes option pricing model requires the variance per period of rate of return on the asset must be estimated. In fact, obtaining such a reliable estimation of the variance is usually very difficult [1]. Furthermore, option pricing model generally assumes that the expected payoffs are characterized by certain probably distributions, geometric Brownian 
motion, for instance. Unfortunately, there does not always exit an efficient market which could justify the assumption on stochastic phenomena. The use of assumption on purely stochastic phenomena may lead to improper investment valuation.

In this paper, we present a comprehensive but simple methodology to evaluate IT investment in a nuclear power station based on real options and fuzzy set theory. Fuzzy risk analysis is able to well formulate the uncertainty of the expected payoffs from an investment, moreover simplifies the real option model in certain degree. This approach will help investors acquire insights about the influence of risk factors on the expected payoff, and then make better valuation and justification of the target investment without complex mathematical calculation.

\section{A Fuzzy Approach to Real Option Valuation}

In this section, we present a new real option approach to value IT investment in nuclear industry. The investment valuation process consists of five stages. In what follows, we explain these steps in details.

Step 1: Risk identification

The first step is to identify the potential risk factors associated with IT investment, and then give a list of the most significant risks. There exist a great variety of risk factors that threaten the success of IT investment. Recently, Benaroch classified the IT investment risks identified by IS research stream into three categories, including firm-specific risks, competition risks and market risks [4].

Step 2: Estimate the risk factors

We assess risk factors largely employing the fuzzy evaluating method present by Ngai [5]. For simplicity of formulation, we adopt triangular fuzzy numbers to characterize the membership function of the linguistic terms. The triangular fuzzy numbers of each linguistic term are set as the same as Ngai does.

Step 3: fuzzy assessment aggregation

Our risk analysis methods allow more than one evaluator to assess the potential risk factors that affect the expected payoffs of an IT investment. After then, the fuzzy average operation is used to balance the deferent judgments from all evaluators. Supposing there are $\mathrm{n}$ evaluators and let $A_{i}=\left(a_{1}{ }^{(i)}, a_{M}{ }^{(i)}, a_{2}{ }^{(i)}\right)$ be the fuzzy numbers, $\mathrm{i}$ $=1, \ldots, \mathrm{n}$. Then, we can get the triangular average mean:

$$
\mathrm{A}_{\text {average }}=\left(m_{1}, m_{M}, m_{2},\right)=\left(\frac{1}{n} \sum_{i=1}^{n} a_{1}{ }^{(i)}, \frac{1}{n} \sum_{i=1}^{n} a_{M}{ }^{(i)}, \frac{1}{n} \sum_{i=1}^{n} a_{2}{ }^{(i)}\right)
$$

After obtaining the fuzzy average of the likelihood and severity of each risk factor, we start to formulate the overall risk level of the IT investment under consideration. Fuzzy weighted average (FWA) is a commonly used operation in risk and decision analysis. To reduce the complexity of comparisons and arithmetic operation, we utilize an efficient fuzzy weighted average algorithm (EFWA) suggested by Lee and Park [6]. 
Step 4: determine expected payoffs and its standard deviation

The result of the calculated fuzzy weighted average is a fuzzy number, which represents the overall risk faced by IT investment under consideration. Since our purpose is to valuate the real option value of the investment, it's required to estimate the expected payoffs and its standard deviation. We calculate the expected payoffs using the following formula:

$$
\mathrm{V}=V^{0}-E\left(V^{0}\right) \bar{R}
$$

Where $V^{0}$ is the initial value of payoffs estimated by decision makers not considering risk factors, $E\left(V^{0}\right)$ denotes the possibilistic mean value of the initial estimation of payoffs. $\mathrm{V}$ is the revised expected payoffs taking into account the probable change range of payoffs caused by risk factors. $V^{0}=\left(V_{1}^{0}, \mathrm{~V}_{M}^{0}, \mathrm{~V}_{2}^{0}\right), \bar{R}=\left(\bar{R}_{1}, \bar{R}_{M}, \bar{R}_{2}\right)$ and $\mathrm{V}=\left(\mathrm{V}_{1}, V_{M}, V_{2}\right)$ are all fuzzy figures.

Supposing $A=\left(a_{1}, a_{M}, a_{2}\right)$ be a triangular fuzzy number then the possibilistic expected value of $\mathrm{A}$ is [7]

$$
\mathrm{E}(A)=\int_{0}^{1} \gamma\left[a_{1}+\gamma\left(a_{M}-a_{1}\right)+a_{2}-\gamma\left(a_{2}-a_{M}\right)\right] d \gamma=\frac{2}{3} a_{M}+\frac{1}{6}\left(a_{1}+a_{2}\right)
$$

Using the addition and scalar multiplication rules for arithmetic operations on triangular fuzzy numbers, we have

$$
\mathrm{V}=\left(\mathrm{V}_{1}, V_{M}, V_{2}\right)=\left(V^{0}{ }_{1}-\mathrm{E}\left(V^{0}\right) \bar{R}_{2}, \mathrm{~V}^{0}{ }_{M}-\mathrm{E}\left(V^{0}\right) \bar{R}_{M}, \mathrm{~V}^{0}{ }_{2}-\mathrm{E}\left(V^{0}\right) \bar{R}_{1}\right)
$$

[7] also introduced the possibilistic variance of fuzzy number $\mathrm{V}$ as

$$
\sigma^{2}(\mathrm{~V})=\frac{1}{2} \int_{0}^{1} \gamma\left[\left(V_{2}-V_{1}\right)(1-\gamma)\right]^{2} d \gamma=\frac{\left(V_{2}-V_{1}\right)^{2}}{24}
$$

Step 5: the real option valuation of the investment

In the last step, we can assess the real option value of the investment based on the result obtained above. For the purpose of simplicity, we assume that only the expected payoff is uncertain and utilize the Black-Scholes pricing model. Then the fuzzy real option value of an investment is [8]

$$
\begin{gathered}
\text { FROV }=\mathrm{VN}\left(d_{1}\right)-X e^{-r T} N\left(d_{2}\right) \\
\text { Where } \mathrm{d}_{1}=\frac{\operatorname{In}(E(V) / X)+\left(r+\sigma^{2} / 2\right) T}{\sigma \sqrt{T}}, \quad d_{2}=d_{1}-\sigma \sqrt{T}
\end{gathered}
$$

Only $\mathrm{V}$ is fuzzy numbers. $\mathrm{E}(\mathrm{V})$ and $\sigma$ represent respectively the possibilistic expected value and the standard deviation of fuzzy figure $\mathrm{V}$. The computing result FROV is also a fuzzy number, representing the real option value of the investment under consideration. 


\section{Numerical Examples}

The Daya Bay Nuclear Power Station, completed in 1996, is located on the coast of the Daya Bay in Guangdong Province, China. In order to maintain efficiency and competency, Daya Bay Nuclear Power Station utilizes information technology applications extensively. The company's Corporate Operation Management Information System (COMIS), has already become an indispensable management platform for its daily operation activities such as preventive maintenance, inventory management, procurement planning, et al.

Recently, the power station plan to establish a supplier relationship management (SRM) system to gain a more reliable supplying source of spares and services it uses. However, valuation of this investment is a challenging task because of uncertainties associated with the investment payoff and cost. In this section, we apply the fuzzy real option valuation approach introduced above to evaluate the SRM investment in Daya Bay Nuclear Power Station. Our purpose is to illustrate how the real option value of an investment could be calculated in fuzzy terms.

To valuate the real option value of the investment, it's required to estimate the expected payoffs and its standard deviation. Therefore, a series of interviews with senior managers in the power station are conducted. The expected payoff come from the SRM investment can be initially estimated as fuzzy triangular figure, which is $V^{0}=\left(V_{1}^{0}, \mathrm{~V}_{M}^{0}, \mathrm{~V}_{2}^{0}\right)=(\$ 376000, \$ 387166, \$ 398332)$. Let $E\left(V^{0}\right)$ denote the possibilistic expected value of fuzzy figure $V^{0}$, then

$$
E\left(V^{0}\right)=\frac{2}{3} \times 387166+\frac{1}{6} \times(376000+398332)=\$ 387,166
$$

Table 1. Mapping of the SRM investment risks and its assessment

\begin{tabular}{ccccc}
\hline \multirow{2}{*}{$\begin{array}{c}\text { Risk } \\
\text { factors }\end{array}$} & \multicolumn{2}{c}{ Evaluator A } & \multicolumn{2}{c}{ Evaluator B } \\
\cline { 2 - 5 } & Likelihood & Severity & Likelihood & Severity \\
\hline \multirow{2}{*}{ Factor } & Medium & High & Medium & Critical \\
1 & $(0.25,0.5,0.75)$ & $(0.5,0.75,1)$ & $(0.25,0.5$, & $(0.75,1,1)$ \\
& & & $0.75)$ & \\
Factor & Medium & Moderate & Unlikely & Moderate \\
2 & $(0.25,0.5,0.75)$ & $(0.25,0.5,0.75)$ & $(0,0.25,0.5)$ & $(0.25,0.5,0.75)$ \\
Factor & Unlikely & Low & Unlikely & Low \\
3 & $(0,0.25,0.5)$ & $(0,0.25,0.5)$ & $(0,0.25,0.5)$ & $(0,0.25,0.5)$ \\
& & & & \\
Factor & Medium & Moderate & Medium & Moderate \\
4 & $(0.25,0.5,0.75)$ & $(0.25,0.5,0.75)$ & $(0.25,0.5,0.75)$ & $(0.25,0.5,0.75)$ \\
& & & & \\
\hline
\end{tabular}


The time horizon of the investment is considered to be 3 years, and $\$ 400,000$ is needed to develop the system. We use $50 \%$ to compute the investment opportunity. There may be a number of risk factors that affect the expected revenue and the deviation of revenues, such as incorrect requirements definition, unfavorable attitudes by users, insufficient experience among team members, or inadequate cooperation of suppliers. Since our purpose is to provide an illustration on calculating process, we simply assume the risks associated with the SRM investment could be mapped as table 1 .

Table 2. Results of fuzzy average of all risk factors

\begin{tabular}{ccc}
\hline Risk factors & $\begin{array}{c}\text { Fuzzy average of } \\
\text { likelihood }\left(W_{i}\right)\end{array}$ & $\begin{array}{l}\text { Fuzzy average of } \\
\text { severity }\left(R_{i}\right)\end{array}$ \\
\hline Factor 1 & $(0.25,0.5,0.75)$ & $(0.625,0.875,1)$ \\
Factor 2 & $(0.125,0.375,0.625)$ & $(0.25,0.5,0.75)$ \\
Factor 3 & $(0,0.25,0.5)$ & $(0,0.25,0.5)$ \\
Factor 4 & $(0.25,0.5,0.75)$ & $(0.25,0.5,0.75)$ \\
\hline
\end{tabular}

The fuzzy averages of each risk factor are showed in table 2. And then, the EFWA algorithm can be used to calculate the overall risk level of the SRM investment. Following the computational procedure defined in EFWA algorithm, we can obtain the resulting membership function of the fuzzy weighted average as $\bar{R}=(0.2222$, $0.5769,0.9167)$. This triangular fuzzy figure represents the overall risk faced by the investment.

Consequently, the revised expected payoffs incorporating the overall risk level can be obtained:

$$
\begin{gathered}
\mathrm{V}=V^{0}-E\left(V^{0}\right) \bar{R}=(\$ 21085, \$ 163810, \$ 312304) \\
E(V)=\frac{2}{3} \times 163810+\frac{1}{6} \times(21085+312304)=\$ 164,771.50
\end{gathered}
$$

The standard deviation of expected payoffs can be calculated as

$$
\sigma(\mathrm{V})=\sqrt{\frac{\left(V_{2}-V_{1}\right)^{2}}{24}}=\sqrt{\frac{(312304-21085)^{2}}{24}}=59444.79
$$

i.e. $\sigma(\mathrm{V})=36 \%$. The last step is to valuate the investment using real option pricing model. We set the other parameters required by Black-Scholes formula as $\mathrm{T}=3, \mathrm{X}=$ $\$ 400,000, r=7 \%$. Then 


$$
\begin{gathered}
\text { FROV }=\mathrm{VN}\left(d_{1}\right)-X e^{-r T} N\left(d_{2}\right) \\
\text { Where } \mathrm{d}_{1}=\frac{\operatorname{In}(164771.5 / 400000)+3\left(0.07+0.36^{2}\right)}{0.36 \sqrt{3}} \\
d_{2}=-0.77382-0.36 \sqrt{3}=-1.39736
\end{gathered}
$$

Thus, we can calculated that the fuzzy value of the real option is $F R O V=(\$ 21684, \$ 9647, \$ 42244)$.

\section{Concluding Remarks}

Real option analysis is a useful tool to formulate the investment decision in uncertain environment, the energy investment, for instance. However, several minor limitations of ROA has prevented its application in practice, even could lead to incorrect valuation. This paper developed a comprehensive but easy-to-use methodology to solve the complicated evaluation problem of ROA.

The valuation approach we present has incorporated fuzzy risk analysis and real option valuation. By utilizing risk analysis based on fuzzy set theory, decision makers could capture the effect of specific risk factors on the expected payoffs, thus obtain a more reliable estimation about parameters of the real option valuation model. It in turn provides a basis for a better evaluating and justifying of the target investment, and avoid complex estimation task at the same time. A numerical example, which involves an SRM investment in a nuclear power station, is presented to illustrate the proposed procedures. We are confident that this method is valuable to help managers produce a well-structured valuation process in information technology investment in nuclear industry. Besides, the approach can be easily applied in the fields of energy economic analyses, such as the evaluation of power plants, building transmission assets, or the adoption of energy efficient technologies.

Acknowledgement. This project is supported by National Natural Science Foundation of China (No. 70571025) and China Postdoctoral Science Foundation (No. 20060400103).

\section{References}

1. Benaroch, M., Kauffman, R.J.: Justifying electronic banking network expansion using real options analysis. MIS Quarterly 24 (2000) 197-225

2. Taudes, A., Feurstein, M., Mild, A.: Options analysis of software platform decisions: a case study. MIS Quarterly 24 (2000) 227- 243

3. Miller, L., Choi, S.H., Park, C.S.: Using an options approach to evaluate Korean information technology infrastructure. The Engineering Economist 49 (2004) 199-219

4. Benaroch, M.: Managing Information Technology Investment Risk: A Real Options Perspective. Journal of Management Information Systems 19 (2002) 43-84 
5. Ngai, E.W.T., Wat, F.K.T.: Fuzzy decision support system for risk analysis in e-commerce development. Decision Support Systems 40 (2005) 235-255

6. Lee, D.H., Park, D.: An efficient algorithm for fuzzy weighted average. Fuzzy Sets and Systems 87 (1997) 39-45

7. Carlsson, C., Fuller, R.: On possibilistic mean value and variance of fuzzy numbers. Fuzzy Sets and Systems 122 (2001) 315-326

8. Carlsson, C., Fuller, R.: A fuzzy approach to real option valuation. Fuzzy sets and systems 139 (2003) 297-312 